\title{
Peptide Shifter: Enhancing Separation Reproducibility Using Correlated Expression Profiles
}

\author{
Dmitri Sitnikov, Joanna M. Hunter, Clive Hayward, Kevin Eng, \\ Isabelle Migneault, Sylvain Tessier, Gregory J. Opiteck, \\ and Paul Kearney \\ Caprion Proteomics, Montreal, Quebec, Canada
}

Chromatographic protein and peptide separation technologies enable comprehensive proteomic analysis of plasma and other complex biological samples by mass spectrometry. However, as the number of separations and/or fractions increases, so does the number of peptides split across fraction boundaries. Irreproducibility of peptide chromatographic separation results in peptides on or near the boundary moving partially or entirely into adjacent fractions. Peptide shifting across fraction boundaries increases the variability of measured peptide abundance, and so there is a trade-off between proteomic comprehensiveness using separation technologies and accurate quantitative proteomic measurements. In this paper, a method for detecting and correcting split peptides, called Peptide Shifter, is introduced and evaluated. An essential component of Peptide Shifter is a global peptide expression profile analysis that allows the inference of the underlying peptide shift pattern without the use of peptide labeling or internal standards. A controlled proteomic analysis of plasma samples demonstrates a $34 \%$ decrease in peptide intensity variability after the application of Peptide Shifter. (J Am Soc Mass Spectrom 2007, 18, 1638-1645) (C) 2007 American Society for Mass Spectrometry

$\mathrm{T}$ The chromatographic separation of biological samples into discrete fractions has become an essential component in the toolbox of proteomic techniques for reducing sample complexity. This is illustrated by the HUPO (Human Proteome Organization) Plasma Proteome Project's (PPP) endeavor to catalogue all the known plasma proteins. Eighteen laboratories participated in the PPP, resulting in over 3000 high-confidence protein identifications [1, 2]. More than 200 protein identifications were generated per sample using multidimensional peptide or protein separation (e.g., SCX-RPLC-MS) before analysis by mass spectrometry [3]. In contrast, laboratories using onedimensional peptide separation (RPLC-MS) identified significantly fewer proteins. Almost all participating laboratories used some form of protein and/or peptide separation, which illustrates the wide adoption of separation technologies in proteomics. The advantages of sample simplification are the same for biomarker discovery studies. Such studies rely on the accuracy of expression profiling and therefore require reproducible sample separation techniques.

Proteomic expression profiling is the comparative analysis of samples to discover differentially expressed

Address reprint requests to Dmitri Sitnikov, Caprion Pharmaceuticals, Protein Analysis, 7150 Alexander Fleming, St-Laurent, Quebec, H4S2C8 Canada. E-mail: dsitnikov@caprion.com proteins among healthy, diseased, or drug-treated populations. Proteomic expression profiling technologies rely on a variety of labeled [4-6] and unlabeled approaches [7-11], where accuracy is enabled by low analytical variability and comprehensiveness is enabled by the extent of separation [12, 13]. Separation technologies that collect fractions in discrete aliquots for subsequent mass spectrometric analysis result in some peptides that elute across multiple fractions. Moreover, as the number of fractions increases, so does the number of peptides contiguously eluting through consecutive fractions [14-16]. We refer to these peptides as split peptides.

As with all methods that transform continuous into discrete signal, there is generally a loss of information in the fractionation process analogous to monitoring chromatography profiles at low sampling rates [17]. This loss of information is illustrated in Figure 1. A peptide completely eluting in one fraction is an unsplit peptide whose elution volume is above the detection limit of a detector. If a peptide is split into two sufficiently large volumes, signal will be detected in both fractions. However, if the split peptide volumes appear at the level below the limit of detection in one of the fractions, then the determination of peptide amount is underestimated (partially degenerate split peptide). Finally, if both split peptide volumes are below the level of detection in both 


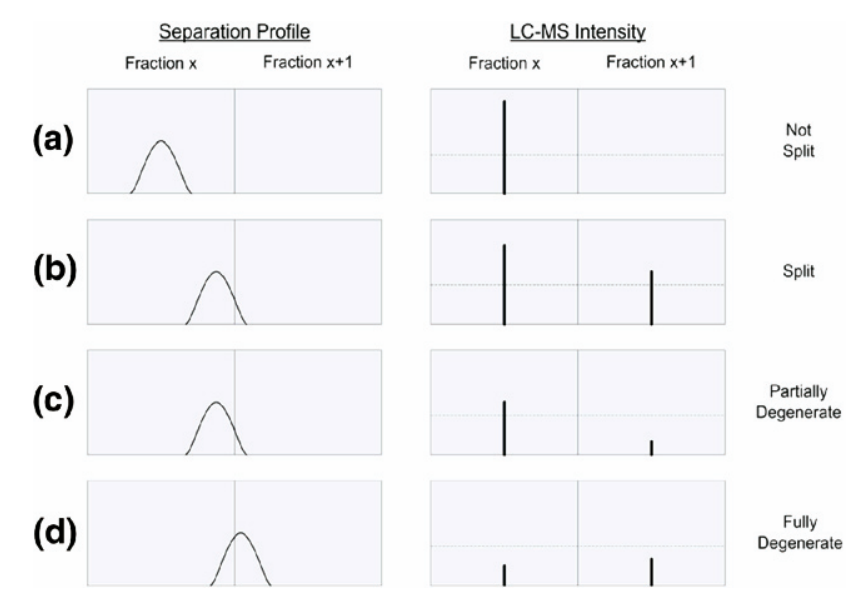

Figure 1. The taxonomy of split peptides. In the Separation Profile pane, the vertical line represents a separation boundary between adjacent chromatographic fractions. In the LC-MS Intensity pane, the horizontal line is the limit of detection. Four types of split peptides are shown (a-d). Peptides that are not split across the boundary fully elute in one fraction (a). Peptides that are split across adjoining fractions and detected by LC-MS in both are called split peptides (b). Partially degenerate split peptides are split across fractions but are below the level of detection in one of the fractions (c). Fully degenerate split peptides are split across fractions but are below the level of detection in both fractions (d).

fractions (fully degenerate) then there is total loss of information. Thus, irreproducible splitting of peptides arising from irreproducible separation and/or fractionation ultimately increases the variability in peptide abundance measurements, and thus degrades the accuracy of expression profiling. The influence of the chromatographic retention time (RT) reproducibility in the first dimension on the reproducibility of MS intensities has, up to now, not received sufficient attention. Based on our experience, the largest inter-run RT shifts observed in our strong cation-exchange (SCX) system vary within $6 \mathrm{~s}$.
For a fraction with elution length of $90 \mathrm{~s}$, this implies that up to $10 \%$ of peptides could be affected by RT shifts at the start of the fraction and another $10 \%$ of peptides affected by RT shifts at the end of the fraction, for a total of $20 \%$ of peptides affected. Therefore it is of practical interest to estimate the influence of these RT shift values on the reproducibility of RPLC-MS profiles.

In expression profiling studies, many biological replicates are analyzed [14], and processing parameters are held constant from replicate to replicate to minimize bias. If separation is performed, aliquots of all samples are collected at fixed time points for subsequent mass spectrometric analysis. Variability in fractionation from sample to sample results in differential peptide splitting across samples in the study. We refer to this as fraction shift and the relative magnitude and direction of fraction shift across samples as the fraction shift profile. These concepts are illustrated in Figure 2. Ideally, split peptides could be detected and then corrected to reduce or eliminate fraction shifting. The most straightforward and accurate approach for the detection and correction of fraction shift would be the use of internal standards, such as isotope-labeled peptides [10]. However, the application of such a method incurs increased costs, sample handling, limits on the number of samples analyzed, and sample complexity [3]. This paper introduces Peptide Shifter, a method for reliable detection and correction of split peptides across samples without the use of internal standards. The method uses a robust technique for inferring the fraction shift profile across samples in a study. A controlled experiment using human plasma samples was performed to measure the impact of fraction shifting on analytical variability and the ability of Peptide Shifter to detect and correct this variability.
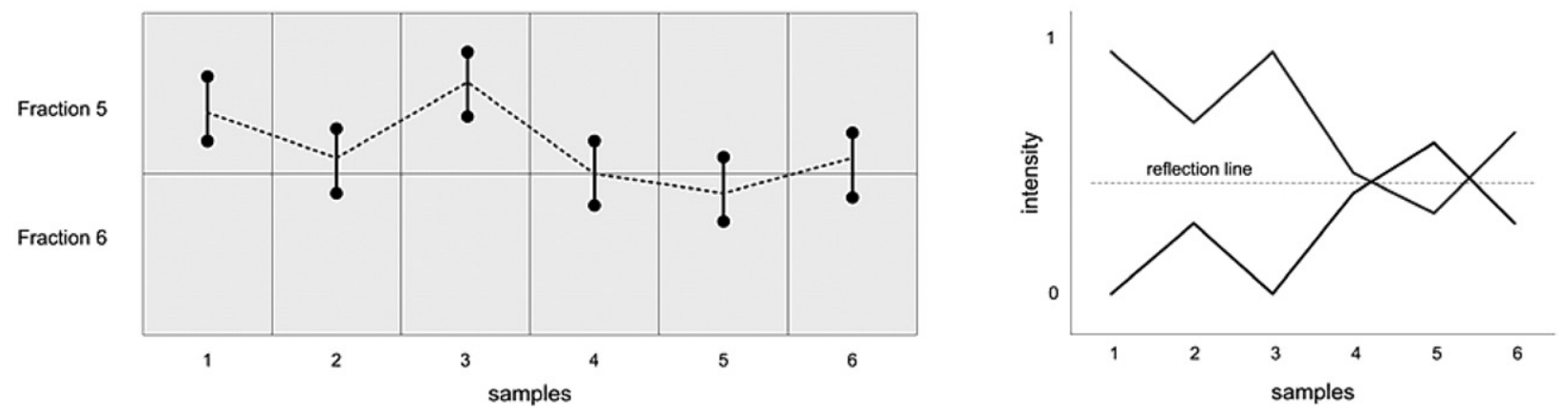

Figure 2. An illustration of a fraction shift profile and the concept of negative correlation. The left panel represents the elution profile of the same peptide in six different SCX runs. The horizontal axis represents the six samples and the vertical axis represents elution time. Each of the vertical bars represents the time span over which the peptide elutes. In this example, the peptide elutes the earliest in Sample 3 and the latest in Sample 5. The dotted line represents the fraction shift pattern across the six samples. The right panel depicts the normalized intensity profile of the peptide in Fractions 5 and 6. The horizontal axis represents the six samples and the vertical axis representss normalized peptide intensity. Because of the conservation of peptide abundance, the sum of the two intensity profiles should be constant. Consequently, the intensity profiles are mirror images of each other. 


\section{Experimental}

\section{Sample Preparation}

Pooled human plasma samples were depleted using MARS (Agilent Technologies, Palo Alto, CA) and digested with Lys-C/Trypsin. After digestion, samples were desalted on HP1100 chromatograph (Agilent Technologies) using C18 guard cartridge (Phenomenex, Torrance, CA). Samples were pooled together and freeze-dried. For strong cation-exchange (SCX) chromatography buffers used for the study consisted of $15 \%$ acetonitrile and 5 or $1000 \mathrm{mM}$ ammonium formate $(\mathrm{pH}$ 3.15 by formic acid) for Buffers A and B, respectively. The stability of the gradient and buffers over the course of six SCX runs was ensured by monitoring the retention time (RT) of standards. Inter-run RT shifts observed in this experiment were random and did not exceed $0.5 \mathrm{~s}$. Samples were dissolved in SCX buffer A and distributed into six aliquots of equal volumes for SCX chromatography. Ninety percent of the volume of each aliquot was injected and separated at $0.8 \mathrm{~mL} / \mathrm{min}$ flow rate using a strong cation exchange $4.6 \times 150 \mathrm{~mm}$, $5 \mu \mathrm{m}$ Biobasic SCX-300 column (Thermo Fisher Scientific, Waltham, MA). The gradient consisted of three linear slopes: 30 to $80 \mathrm{mM}$ for $6 \mathrm{~min}, 80$ to $150 \mathrm{mM}$ for $4 \mathrm{~min}, 150$ to $750 \mathrm{mM}$ for $4 \mathrm{~min}$, followed by isocratic gradient at $750 \mathrm{mM}$ for $2 \mathrm{~min}$.

Each lyophilized SCX fraction was re-solubilized in $90 / 10$ water/acetonitrile, $0.2 \%$ trifluoroacetic acid (TFA) before LC-MS analysis. The reversed-phase (RP) LC-MS system consisted of a CapLC pump and a Q-TOF Ultima mass spectrometer (Waters MicroMass UK, Manchester, UK). Peptide separation was achieved using a home-packed Jupiter 300 C18 column $(150 \mu \mathrm{m} \times$ $10 \mathrm{~cm}$, stationary phase from Phenomenex). A linear gradient of $10-40 \%$ acetonitrile $(0.2 \%$ formic acid) in 65 min was executed at a flow rate of $12.5 \mu \mathrm{L} / \mathrm{min}$.

\section{Experimental Design}

An uninterrupted sequence of six runs with fractionation (eight unequal fractions covered the range of 13 min) was executed. To mimic RT shifts the fraction collection was shifted uniformly (so neither the length of individual fractions nor the total length of fractionation was changed). Therefore, the second triplet of samples (Group 2) was run with the same gradient, but the fractionation was shifted $12 \mathrm{~s}$ toward the beginning of the run comparatively to the first triplet (designated as Group 1). The fractionation rather than the gradient was shifted to simplify the execution and analysis of the experiment. The maximum RT variations were $<1 \mathrm{~s}$.

\section{Peptide Detection}

The LC-MS data were smoothed in both the $\mathrm{m} / \mathrm{z}$ and retention time dimensions, baseline subtracted, and centroided. An isotopic model was applied to all local maxima to determine the monoisotopic peaks. For each resulting peak $\mathrm{m} / \mathrm{z}$, retention time, charge, and intensity were reported.

\section{Normalization and Clustering}

To minimize analytic variability caused by LC-MS analysis, the three dimensions of LC-MS data (mass, retention time, intensity) were normalized. For each fraction, a standard sample was selected and all other samples aligned to it on mass and retention time coordinates. A dynamic and nonlinear algorithm was used to correct the retention time domain of each sample to the standard sample. After the correction of retention times, all pairs of aligned peptides were used to derive a normalization factor to adjust sample intensities to the standard sample intensity domain. Peptides were then clustered on similar mass and retention time using standard hierarchical clustering methods. For each peptide cluster, a median mass and retention time were used to represent peptides in the cluster. This results in regression to the norm, further increasing the accuracy of mass and retention time. Normalized peptide clusters reproducibly matched in three or more runs out of six were selected for the analysis. This level of reproducibility is routinely applied to eliminate low-confidence peptides.

\section{Detection of Nondegenerate Split Peptides}

Pairs of peptide clusters from adjoining fractions are matched that have the same $m / z$, retention time, and charge values. Matching tolerances for $m / z$ and retention time were set to $0.1 \mathrm{Da}$ and $1 \mathrm{~min}$, respectively. Furthermore, the expression profiles of the peptide cluster pairs must be negatively correlated with Pearson correlation coefficient less than -0.20 .

\section{Detection of Degenerate Split Peptides}

By definition, a degenerate split peptide is not detected in adjoining fractions, and thus the fraction shift profile (which is the fingerprint of split peptides) is found first and then putative degenerate split peptides are correlated to the fraction shift profile. We describe both a supervised and an unsupervised method for determining the fraction shift profile. The unsupervised method uses high-confidence split peptides to define the fraction shift profile. First, all pairs of peptide clusters in adjoining fractions that match on $\mathrm{m} / \mathrm{z}$, retention, and charge (0.1 Da and $1 \mathrm{~min}$ matching tolerances) are found. Furthermore, these peptide clusters were required to be highly reproducible (found in all six samples) and be strongly and negatively correlated with Pearson score below -0.75 . From these peptide clusters a consensus expression profile is obtained by taking the median normalized intensity value in each sample. This consensus expression profile becomes the fraction shift profile. 
The supervised method applies hierarchical clustering with average linkage and Spearman correlation to all normalized peptide cluster expression profiles in a fraction. The most prevalent expression profiles are reported for each fraction along with their median consensus profile. The most common consensus expression profile across all fractions is selected as the fraction shift profile.

Normally, the supervised method for finding the fraction shift profile is used. However, if the investigator has auxiliary data such as UV profile data from the SCX separation runs then these can be combined with the supervised or unsupervised method to enhance confidence in the fraction shift profile.

Once the fraction shift profile has been determined, all peptide clusters highly correlated to the fraction shift profile are selected. Specifically, peptide clusters with a Pearson correlation score above 0.70 or below -0.70 are selected.

\section{Correction of Peptide Intensities}

The expression profiles for matched nondegenerate split peptides from adjoining fractions were added together to form the new integrated profile. Expression profiles for each degenerate split peptide were added to the fraction shift profile pattern (normalized to the median intensity of the degenerate split peptide). The new integrated profiles replace the original profiles.

\section{Data Management}

All data are captured in a peptide database where each peptide is defined along the following dimensions. A study consists of replicates grouped into conditions. For example, the fraction shift experiment has two conditions ("No Shift" and "Shifted") and three replicates in each condition. Each peptide cluster found in a study is assigned a unique cluster identifier. Furthermore, each member of a peptide cluster has an observed fraction, $\mathrm{m} / \mathrm{z}$, retention time, charge, and intensity. Peptide cluster updates such as expression profile corrections are tracked in the database.

\section{Results and Discussion}

In the fraction shift experiment, each peptide cluster was classified as being nonsplit, split, or degenerate using supervised fraction shift analysis. Out of 27,288 peptide clusters, a total of $20 \%$ were affected by the forced fraction shift of 12 s: $2 \%$ of peptides were split peptides and $18 \%$ were degenerate (either totally or partially) split peptides. The larger number of degenerate split peptides is consistent with expectations for two reasons. First, the SCX elution profile of a peptide has a long tail to the right (see Figure 1), and thus the fraction boundary will likely intersect the tail of a peptide's elution profile. Furthermore, if the fraction boundary intersects the tail of a peptide's elution profile, then likely the resulting LC-MS peptide intensity measurement for the tail portion will be below the LC-MS detection limit. Second, approximately half of all peptides have intensity less than threefold the LC-MS detection limit. Low-intensity peptides are frequently degenerate because, when they are split, at least one of the halves is likely to be below the LC-MS detection limit. The combination of these two factors increases the number of degenerate split peptides above nondegenerate split peptides.

Examples of peptides detected and corrected by Peptide Shifter appear in Figure 3. In the top left panel of Figure 3, an example of a nondegenerate split peptide is shown. Notice how the peptide profiles of the matched peptide clusters are mirror images of each other. An example of a partially degenerate split peptide located on the border between Fractions 1 and 2 can be seen in the lower right panel of Figure 3. This peptide was detected in Fraction 1 only before fraction shift and detected in Fraction 2 only after the fraction shift. Despite being partially degenerate, the total peptide intensity has low variability.

To quantify the improvement of split peptide correction, the coefficient of variation $(\mathrm{CV})$ of peptide intensity before and after correction was determined for split peptides and for all peptides. For the set of peptides identified as split peptides, the $\mathrm{CV}$ of peptide intensity before correction was $32 \%$ and after correction was $16 \%$. For the set of peptides identified as degenerate split peptides, the $\mathrm{CV}$ of peptide intensity before correction was $40 \%$ and after correction decreased to $14 \%$. These values agree with the $C V$ of peptide intensities of technical LC-MS repeats $(\sim 14 \%)$ that is obtained for nonshifted samples (data not shown). In addition, the analysis of the intensity variability for all peptides shows that the CV decreases from 29 to $19 \%$ after fraction shift correction. Thus, Peptide Shifter reduces overall peptide intensity variability by $34 \%$.

Intensities of split peptides are negatively correlated as can be seen in Figure 4. Peptides randomly selected from adjacent fractions had their expression profiles correlated. Because these peptides are not biased to being split peptides, their Pearson correlation scores are centered around zero, as seen in the distribution on the left. Pairs of peptide clusters matched between adjoining fractions on $m / z$, retention time, and charge (thus biased to being split peptides) have a negatively skewed distribution of Pearson correlation scores. This analysis supports the approach of using expression profile correlation to detect split peptides.

The supervised learning method was applied to all eight fractions of all six SCX runs. The resulting fraction shift profiles were clustered into the six most frequently occurring patterns (depicted in Figure 5). The upper panel on the left demonstrates a pattern where peptide intensities reproducibly increase in the shifted runs. The inverse pattern can be seen in the middle upper panel. These two shift patterns correspond to peptides shifting in and shifting out of Fraction 2 because of their 

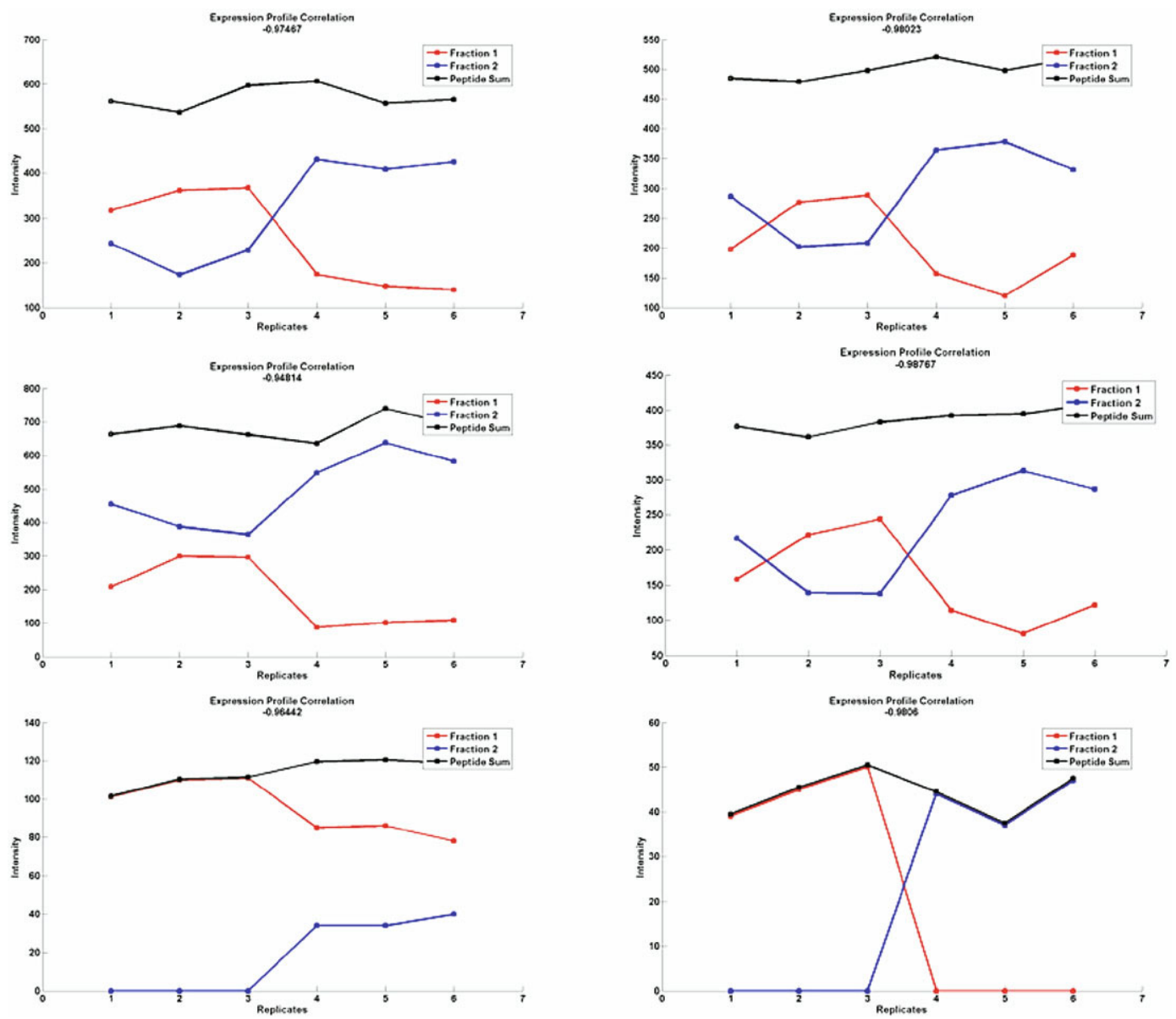

Figure 3. Examples of detection and correction of split peptide. The red and blue expression profiles correspond to a pair of matched peptides from Fractions 1 and 2, respectively. The corrected profile appears in black. As expected, there is an intensity differential between the first three replicates (Group 1, no shift applied) and the last three replicates (Group 2, controlled shift) due to peptide splitting.

Correlation Distribution for Random Peptides

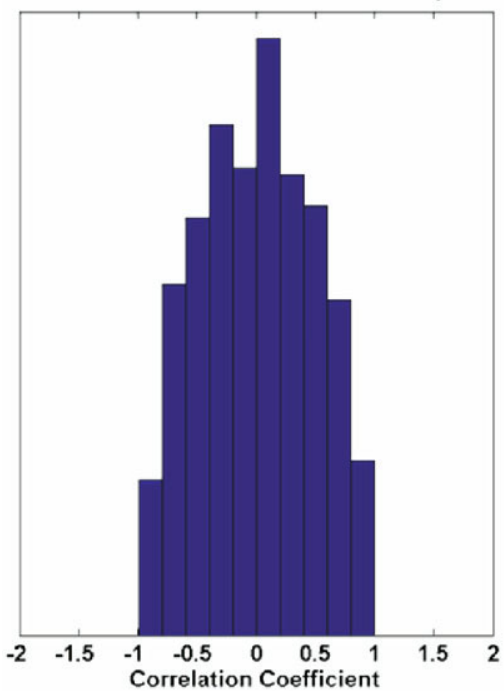

Correlation Distribution for Matched Peptides

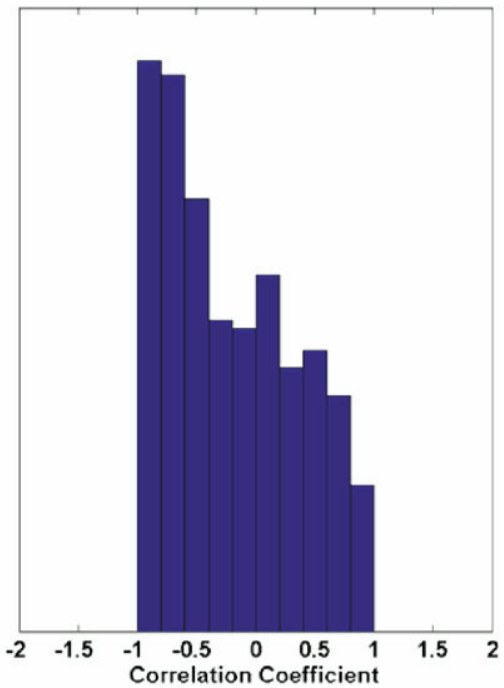

Figure 4. Distribution of correlation coefficients of peptides from adjacent fractions. In both distributions peptides are selected from adjoining fractions. On the left, peptide pairs are selected randomly, whereas on the right peptides are matched on $\mathrm{m} / \mathrm{z}$, retention time and charge. The distribution on the right is biased to negatively correlated peptides. 

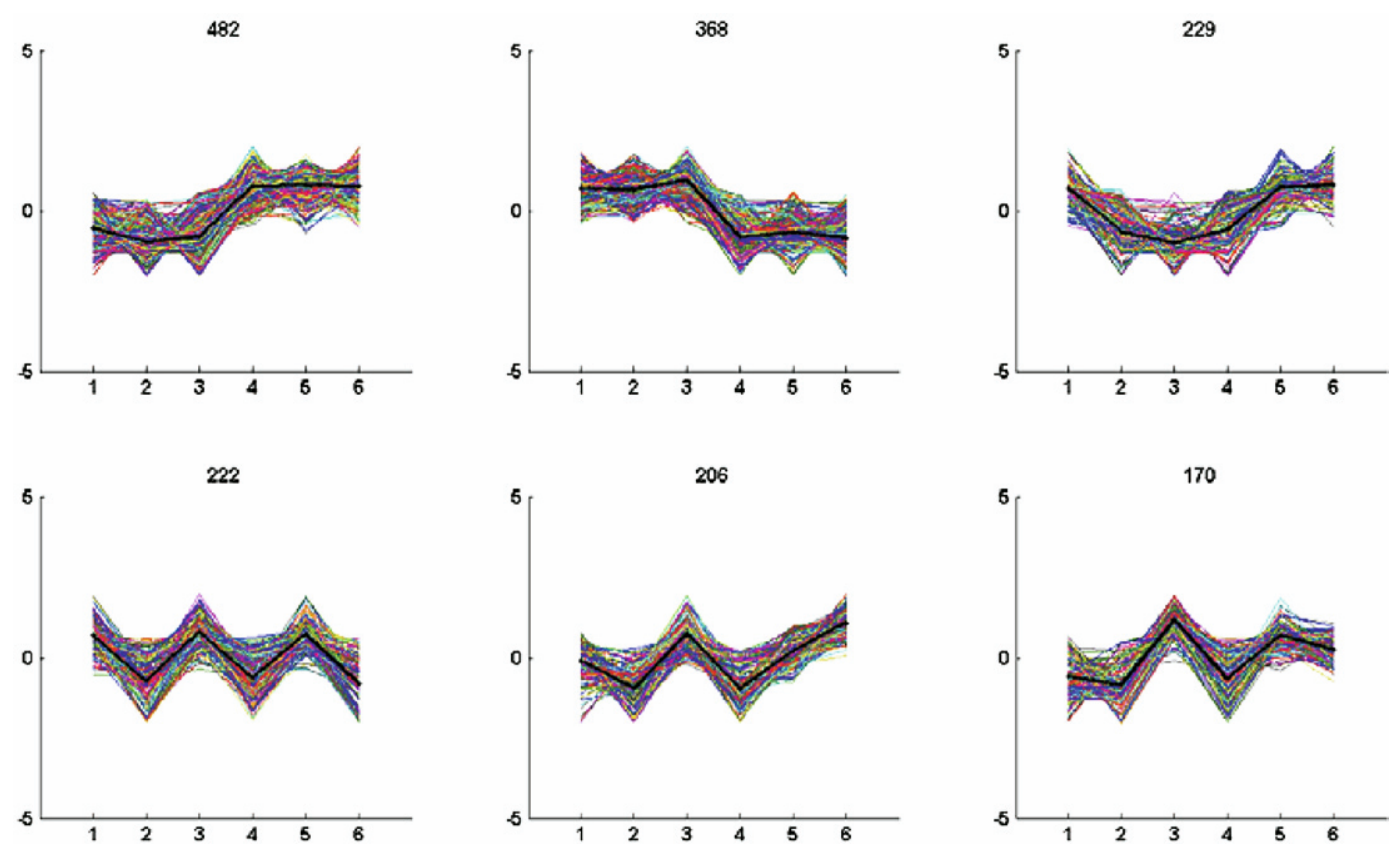

Figure 5. The most frequently occurring expression profile patterns in Fraction 2. The number above each plot indicates the number of peptide clusters associated with that pattern. The two most common expression profiles are consistent with the expected fraction shift profile for the controlled experiment.

position on the front and back borders of Fraction 2, respectively. These two patterns are the most prevalent expression profiles, as expected, by design of the controlled fraction shift experiment. These two expected patterns are among the three most prevalent expression profiles in six of the eight fractions. For the remaining two fractions they are among the four most prevalent patterns. It is interesting to note that the relative

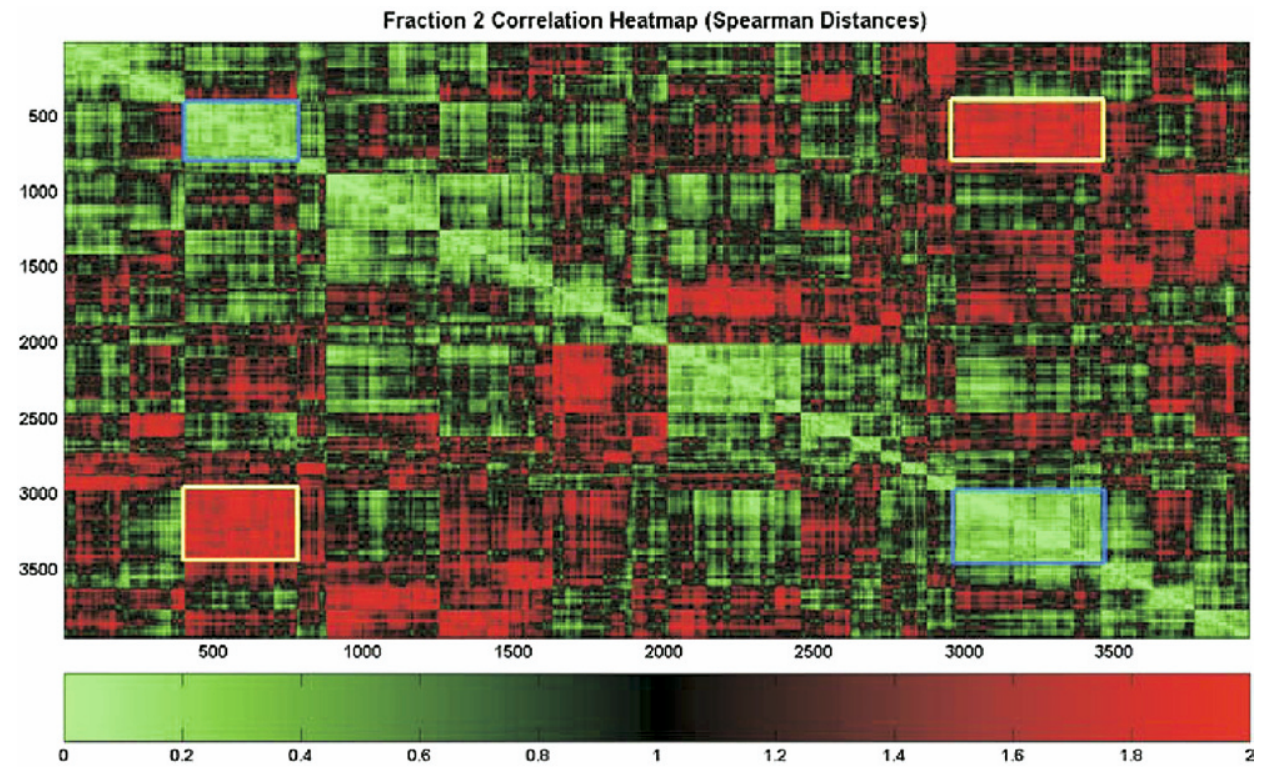

Figure 6. Expression profile correlation heatmap for all pairs of peptides from Fraction 2. Each point in the heatmap represents the Spearman correlation between the expression profiles of two peptides. Green is high correlation and red is low correlation. Peptides are highly correlated to themselvesthus, the diagonal green line. Hierarchical clustering has been applied to group highly correlated peptides. Framed in blue are the peptides corresponding to the two most frequently occurring expression profiles in Fraction 2, which appear in Figure 5. The upper left blue box corresponds to peptides shifted into Fraction 2, whereas the lower right blue box corresponds to peptides shifted out of Fraction 2. As expected, these two sets of peptides are highly negatively correlated (two yellow boxes). 
changes in intensities of peptides entering Fraction 2 from Fraction 1 and leaving from Fraction 2 to Fraction 3 were similar and correlate. Consequently, the supervised approach determined the expected fraction shift profile.

The correlation of relative changes in intensities of peptides on opposite borders is illustrated in Figure 6, which depicts the results of hierarchical clustering of all Fraction 2 peptides using average linkage and Spearman correlation. Expression profiles for peptides located at the front border of fraction two (upper left, blue box) are highly positively correlated (i.e., green). Similarly, expression profiles for peptides located at the back border of fraction two (lower right, blue box) are positively correlated. Consequently, split peptides are among the most positively correlated peptides. Furthermore, those peptides shifted into Fraction 2 and those shifted out of Fraction 2 are strongly negatively correlated (upper right and lower left, yellow boxes), as expected.

Unsupervised learning, as defined in the Experimental section, selected 65 peptides, and these are depicted in Figure 7. The expression profiles of these 65 peptides share the pattern consistent with the design of the fraction shift experiment. Note that it is advisable to apply the supervised and unsupervised methodologies in parallel to boost confidence in the determination of the fraction shift pattern. In this analysis, the two methods yield the same result.

To confirm the ability of Peptide Shifter to detect fraction shifts in the context of a real study, it was applied to the proteomic analysis of 104 human plasma samples fractionated by SCX over 6 consecutive days (Figure 8). The Generation of Fraction Shift Profile was based on 273 peptides using the unsupervised Peptide Shifter method. Twenty randomized permutation tests were applied to the LC-MS intensity data to estimate the probability that these 273 peptides could be obtained by chance alone. The 20 permutation tests aver-

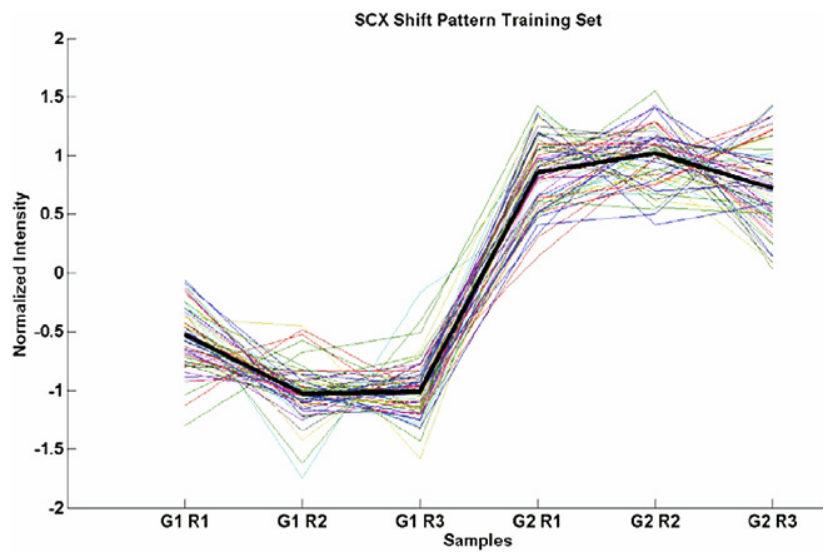

Figure 7. Expression profile derived from the 65 peptides with the most negatively correlated matches in adjoining bands. The horizontal axis are three replicates of Group 1 followed by the three replicates of Group 2.

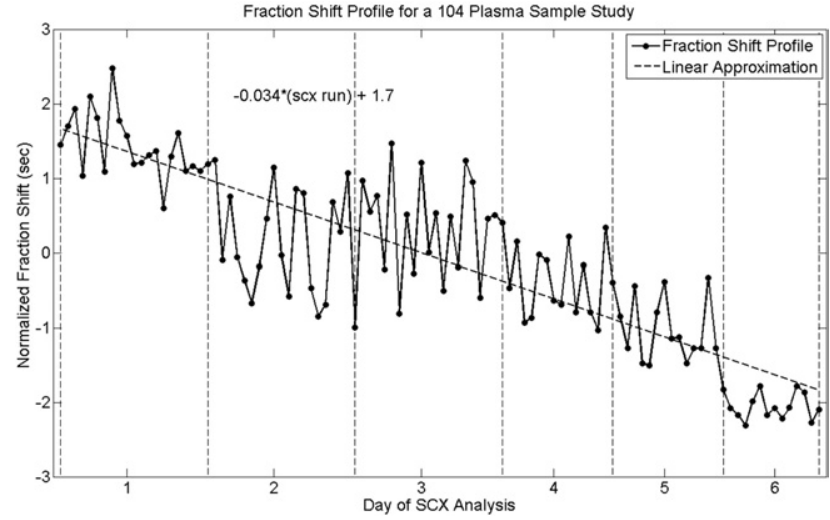

Figure 8. Fraction shift profile analysis of a study consisting of 104 plasma samples. The samples were processed sequentially by SCX over 6 days resulting in 8 fractions per sample. Each sample fraction was then analyzed by LC-MS using the same protocols described in the Experimental section. Peptide Shifter was applied to this dataset to first determine the Fraction Shift Profile and then to detect split peptides.

aged 18 peptides, resulting in a false positive rate of $7 \%$. Because this was a large-scale proteomic study, several aspects of fraction shifting are apparent that could not be seen in the controlled study on six samples. First, the downward trending of the profile is evident (Figure 8). A linear fit for this trending was obtained and calibrated against UV profiles for the SCX runs to establish an absolute scale for fraction shift in seconds ( $y$-axis). The magnitude of the largest fraction shift over the 104 SCX runs was about $6 \mathrm{~s}$. This enables quantification of between-run variability as well as between-day variability. The downward trending is likely the result of column aging. Also apparent is that Day 2 and Day 6 had different behaviors than did the other days, indicating that there are other factors contributing to fraction shifting. Peptide Shifter determined that 3313 out of 44,070 (7.5\%) peptides were split. Median CV for nonsplit and split peptides was estimated as 38 and $54 \%$, respectively. After normalization of split peptides, their median CV was decreased to $41 \%$.

\section{Conclusion}

For the SCX gradient presented here, Peptide Shifter reduces peptide intensity variation by detecting and correcting split peptides. Overall, peptide intensity variability was reduced $34 \%$. Peptides identified as being split had peptide intensity variability reduced by $64 \%$.

In addition to reducing peptide intensity variability, Peptide Shifter provides diagnostic information throughout the gradient of the separation run. Although the fraction shift profile discovered in this experiment was consistent from fraction to fraction (by design of the experiment), Peptide Shifter detects the fraction shift profile on a per fraction basis. Thus, nonuniform fraction shifts can be detected and corrected using Peptide Shifter as well as the uniform fraction shift analyzed 
herein. Peptide Shifter also enables the diagnosis and quantification of column aging and the identification of outlier SCX runs.

The limits of Peptide Shifter have not been completely examined. In particular, the performance of Peptide Shifter as the number of fractions increases is unknown. There are at least two issues to be addressed as the number of fractions increases. First, as the number of fractions increases, the number of peptides split across three or more fractions also increases. Peptide Shifter can be extended to detect and correct these peptides. Specifically, peptide matching can be performed across multiple contiguous fractions to determine the start and end fractions of a peptide's elution. Negative correlation and fraction shift learning techniques still apply. Second, as the number of fractions increases, the number of low-intensity peptides increases disproportionately. Thus, the relative proportion of degenerate split peptides to nondegenerate split peptides will increase. The performance of Peptide Shifter under these conditions is unknown.

It is important to note that confident detection and correction of partially degenerate split peptides will decrease if the number of samples is very small (i.e., four or fewer). We plan to extend the utility of Peptide Shifter to make it applicable to very small studies.

Peptide Shifter has been successfully applied to several in-house clinical studies where the large number of samples required, for example, multiple columns, multiple days of processing, multiple operators, and multiple batches of buffer. These results will be the subject of a subsequent publication.

\section{References}

1. Omenn, G. S.; States, D. J.; Adamski, M.; Blackwell, T. W.; Menon, R.; Hermjakob, H.; Apweiler, R.; Haab, B. B.; Simpson, R. J.; Eddes, J. S.; Kapp, E. A.; Moritz, R. L.; Chan, D. W.; Rai, A. J.; Admon, A.; Aebersold, R.; Eng, J.; Hancock, W. S.; Hefta, S. A.; Meyer, H.; Paik, Y. K.; Yoo, J. S.; Ping, P.; Pounds, J.; Adkins, J.; Qian, X.; Wang, R.; Wasinger, V.; Wu, C. Y.; Zhao, X.; Zeng, R.; Archakov, A.; Tsugita, A.; Beer, I.; Pandey, A.;
Pisano, M.; Andrews, P.; Tammen, H.; Speicher, D. W.; Hanash, S. M. Overview of the HUPO Plasma Proteome Project: Results from the Pilot Phase with 35 Collaborating Laboratories and Multiple Analytical Groups, Generating a Core Dataset of 3020 Proteins and a Publicly-Available Database. Proteomics 2005, 5, 3226-3245.

2. Adamski, M.; Blackwell, T.; Menon, R.; Martens, L.; Hermjakob, H.; Taylor, C.; Omenn, G. S.; States, D. J. Data management and preliminary data analysis in the pilot phase of the HUPO Plasma Proteome Project. Proteomics 2005, 5, 3246-3261.

3. Washburn, M. P.; Wolters, D.; Yates, J. R., 3rd. Large-scale analysis of the yeast proteome by multidimensional protein identification technology. Nat. Biotechnol. 2001, 19, 242-247.

4. Faber, M. J.; Agnetti, G.; Bezstarosti, K.; Lankhuizen, I. M.; Dalinghaus, M.; Guarnieri, C.; Caldarera, C. M.; Helbing, W. A.; Lamers, J. M. Recent developments in proteomics: implications for the study of cardiac hypertrophy and failure. Cell. Biochem. Biophys. 2006, 44, 11-29.

5. Aggarwal, K.; Choe, L. H.; Lee, K. H. Shotgun proteomics using the iTRAQ isobaric tags. Brief Funct. Genomic. Proteomic 2006, 5, 112-120.

6. Han, D. K.; Eng, J.; Zhou, H.; Aebersold, R. Quantitative profiling of differentiation-induced microsomal proteins using isotope-coded affinity tags and mass spectrometry. Nat. Biotechnol. 2001, 19, 946-951.

7. Kantor, A. B.; Wang, W.; Lin, H.; Govindarajan, H.; Anderle, M.; Perrone, A.; Becker, C. Biomarker discovery by comprehensive phenotyping for autoimmune diseases. Clin. Immunol. 2004, 111, 186-195.

8. Old, W. M.; Meyer-Arendt, K.; Aveline-Wolf, L.; Pierce, K. G.; Mendoza, A.; Sevinsky, J. R.; Resing, K. A.; Ahn, N. G. Comparison of label-free methods for quantifying human proteins by shotgun proteomics. Mol. Cell. Proteomics 2005, 4, 1487-1502.

9. Silva, J. C.; Denny, R.; Dorschel, C.; Gorenstein, M. V.; Li, G. Z.; Richardson, K.; Wall, D.; Geromanos, S. J. Simultaneous qualitative and quantitative analysis of the Escherichia coli proteome: a sweet tale. Mol. Cell. Proteomics 2006, 5, 589-607.

10. Silva, J. C.; Denny, R.; Dorschel, C. A.; Gorenstein, M.; Kass, I. J.; Li, G. Z.; McKenna, T.; Nold, M. J.; Richardson, K.; Young, P.; Geromanos, S. Quantitative proteomic analysis by accurate mass retention time pairs. Anal. Chem. 2005, 77, 2187-2200.

11. Silva, J. C.; Gorenstein, M. V.; Li, G. Z.; Vissers, J. P.; Geromanos, S. J. Absolute quantification of proteins by LCMSE: a virtue of parallel MS acquisition. Mol. Cell. Proteomics 2006, 5, 144-156.

12. Li, X. J.; Yi, E. C.; Kemp, C. J.; Zhang, H.; Aebersold, R. A software suite for the generation and comparison of peptide arrays from sets of data collected by liquid chromatography-mass spectrometry. Mol. Cell. Proteomics 2005, 4, 1328-1340.

13. Gaspari, M.; Verhoeckx, K. C.; Verheij, E. R.; van der Greef, J. Integration of two-dimensional LC-MS with multivariate statistics for comparative analysis of proteomic samples. Anal. Chem. 2006, 78, 2286-2296.

14. Davis, J. M.; Giddings, J. C. Statistical method for estimation of number of components from single complex chromatograms: application to experimental chromatograms. Anal. Chem. 1985, 57, 2178-2182.

15. Davis, J. M.; Giddings, J. C. Statistical method for estimation of number of components from single complex chromatograms: theory, computerbased testing, and analysis of errors. Anal. Chem. 1985, 57, 2168-2177.

16. Davis, J. M.; Giddings, J. C. Origin and characterization of departures from the statistical model of component-peak overlap in chromatography. J. Chromatogr. 1984, 289, 277-298.

17. Halket, J. M.; Waterman, D.; Przyborowska, A. M.; Patel, R. K.; Fraser P. D.; Bramley, P. M. Chemical derivatization and mass spectral libraries in metabolic profiling by GC/MS and LC/MS/MS. J. Exp. Bot. 2005, 56, 219-243. 